\title{
On formulas for moments of the Wishart distributions as weighted generating functions of matchings
}

\author{
Yasuhide NUMATA ${ }^{1,3}$ and Satoshi KURIKI ${ }^{2,3}$ \\ ${ }^{1}$ Department of Mathematical Informatics, University of Tokyo, 7-3-1 Hongo, Bunkyo-ku, Tokyo 113-0033, Japan. \\ ${ }^{2}$ The Institute of Statistical Mathematics, 10-3 Midoricho, Tachikawa, Tokyo 190-8562, Japan. \\ ${ }^{3}$ Japan Science and Technology Agency (JST), CREST.
}

\begin{abstract}
We consider the real and complex noncentral Wishart distributions. The moments of these distributions are shown to be expressed as weighted generating functions of graphs associated with the Wishart distributions. We give some bijections between sets of graphs related to moments of the real Wishart distribution and the complex noncentral Wishart distribution. By means of the bijections, we see that calculating these moments of a certain class the real Wishart distribution boils down to calculations for the case of complex Wishart distributions.

Résumé. Nous considérons les lois Wishart non-centrale réel et complexe. Les moments sont décrits comme fonctions génératrices de graphes associées avex les lois Wishart. Nous donnons bijections entre ensembles de graphes relatifs aux moments des lois Wishart non-centrale réel et complexe. Au moyen de la bijections, nous voyons que le calcul des moments d'une certaine classe la loi Wishart réel deviennent le calcul de moments de loi Wishart complexes.
\end{abstract}

Keywords: generating funtion; Hafnian; matching; moments formula; Wishart distribution.

\section{Introduction}

First we recall the Wishart distributions which originate from the paper by Wishart [18]. Let $X_{1}=$ $\left(x_{i 1}\right)_{1 \leq i \leq p}, X_{2}=\left(x_{i 2}\right)_{1 \leq i \leq p}, \ldots, X_{\nu}=\left(x_{i \nu}\right)_{1 \leq i \leq p}$ be $p$-dimensional random column vectors distributed independently according to the normal (Gauss) distribution $N_{p}\left(\mu_{1}, \Sigma\right), \ldots, N_{p}\left(\mu_{\nu}, \Sigma\right)$ with mean vectors $\mu_{1}=\left(\mu_{i 1}\right)_{1 \leq i \leq p}, \ldots, \mu_{\nu}=\left(\mu_{i \nu}\right)_{1 \leq i \leq p}$ (respectively) and a common covariance matrix $\Sigma=$ $\left(\sigma_{i j}\right)$. The distribution of a $p \times p$ symmetric random matrix $W=\left(w_{i j}\right)_{1 \leq i, j \leq p}$ defined by $w_{i j}=$ $\sum_{t=1}^{\nu} x_{i t} x_{j t}$ is the real noncentral Wishart distribution $W_{p}(\nu, \Sigma, \Delta)$, where $\Delta=\left(\delta_{i j}\right)_{1 \leq i j \leq p}$ is the mean square matrix defined by $\delta_{i j}=\sum_{t=1}^{\nu} \mu_{i t} \mu_{j t}$. The Wishart distribution for $\Delta=0$ is said to be central and is denoted by $W_{p}(\nu, \Sigma)$.

The matrix $\Omega=\Sigma^{-1} \Delta$ is called the noncentrality matrix. It is usually used instead of $\Delta$ to parameterize the Wishart distribution. However, in this paper, we use $(\nu, \Sigma, \Delta)$ for simplicity in describing formulas. The complex Wishart distribution $C W_{p}(\nu, \Sigma, \Delta)$ is defined as the distribution of some $p \times p$ Hermitian random matrix constructed from random vectors distributed independently according to the complex normal (complex Gauss) distributions. 
The moment generating function of the real Wishart distribution is given by

$$
\mathbb{E}\left[e^{\operatorname{tr}(\Theta W)}\right]=\operatorname{det}(I-2 \Theta \Sigma)^{-\frac{\nu}{2}} e^{-\frac{1}{2} \operatorname{tr}(I-2 \Theta \Sigma)^{-1} \Theta \Delta}
$$

where $\Theta$ is a $p \times p$ symmetric parameter matrix [13]. Similarly, the moment generating function of the complex Wishart distribution is given as follows:

$$
\mathbb{E}\left[e^{\operatorname{tr}(\Theta W)}\right]=\operatorname{det}(I-\Theta \Sigma)^{-\nu} e^{-\operatorname{tr}(I-\Theta \Sigma)^{-1} \Theta \Delta},
$$

where $\Theta$ is a $p \times p$ Hermitian parameter matrix. See also [2] for the central case. Our first objective is to describe the moments $\mathbb{E}\left[w_{i_{1}, i_{2}} w_{i_{3}, i_{4}} \cdots w_{i_{2 n-1}, i_{2 n}}\right]$ of the Wishart distributions of general degrees in explicit forms. Since the Wishart distribution is one of the most important distributions, it has been studied by many researchers not only in the field of mathematical statistics but also in other fields (e.g., [1, 11]). Its moments have been well studied, and methods to calculate the moments in the central cases have been developed by Lu and Richards [10]; Graczyk, Letac, and Massam [3, 4]; Vere-Jones [16]; and many other authors. In particular, Graczyk, Letac and Massam [3, 4] developed a formula for the moments using the representation theory of symmetric group. More recently, Letac and Massam [9] introduced a method to calculate the moments of the noncentral Wishart distributions. In this paper, we introduce another formula for the moments of Wishart distribution; in our formula, the moments are described as special values of the weighted generating function of matchings of graphs. Calculation of the moments boils down to enumeration of graphs via our formulas. As an application of our formulas, we construct some correspondences between some sets of graphs, which implies several identities of moments.

The organization of this paper is as follows. In Section 2, we introduce some notations for the graphs. In Section 3, we define the generating functions of matchings and give the main formulas, which are an extension of Takemura [15] dealing with the central case. In Section 4.1] we give some correspondences between directed and undirected graphs, which implies equations between the moments of the complex Wishart distribution and the moments of the real Wishart distribution for some special parameter. In Sections 4.2 and 4.3, we consider the Wishart distribution with some degenerated parameters. We see that the calculation of its moments is reduced to enumerating graphs satisfying some conditions.

A part of this paper is taken from our previous paper [8]. Please see the paper for the omitted proofs.

\section{Notation of graphs}

In this paper, we consider both undirected and directed graphs. For $l \in \mathbb{Z}$, we define $i$ and $\ddot{l}$ by $\dot{l}=2 l-1$ and $\ddot{l}=2 l$. Let us fix $n \in \mathbb{Z}_{>0}$. We also fix sets $V$ and $V^{\prime}$ as follows: $V=[n]=\{1, \ldots, n\}$, $\dot{V}=[\dot{n}]=\{\dot{1}, \ldots, \dot{n}\}, \ddot{V}=[\ddot{n}]=\{\ddot{1}, \ldots, \ddot{n}\}$, and $V^{\prime}=\dot{V} \amalg \ddot{V}=[\dot{n}] \amalg[\ddot{n}]=[2 n]$. We use $V$ and $V^{\prime}$ as the sets of vertices of directed and undirected graphs, respectively.

First we consider undirected graphs. For $v \neq w$, the undirected edge between $v$ and $w$ is denoted by $\{v, w\}=\{w, v\}$. We do not consider undirected self loops, i.e., $\{v, v\}$. For sets $W^{\prime}$ and $U^{\prime}$ of vertices, we define sets $K_{U^{\prime}}^{\prime}$ and $K_{W^{\prime}, U^{\prime}}^{\prime}$ of undirected edges by $K_{W^{\prime}, U^{\prime}}^{\prime}=\left\{\{w, u\} \mid w \in W^{\prime}, u \in U^{\prime}, w \neq u\right\}$, $K_{U^{\prime}}^{\prime}=K_{U^{\prime}, U^{\prime}}^{\prime}=\left\{\{v, u\} \mid v \neq u \in V^{\prime}\right\}$. We call a pair $G^{\prime}=\left(V^{\prime}, E^{\prime}\right)$ of a finite set $V^{\prime}$ and a subset $E^{\prime} \subset K_{V^{\prime}}^{\prime}$ an undirected graph. For an undirected graph $G^{\prime}=\left(V^{\prime}, E^{\prime}\right)$, we define vertex $\left(E^{\prime}\right)$ by $\operatorname{vertex}\left(E^{\prime}\right)=\left\{v \in V^{\prime} \mid\{v, u\} \in E^{\prime}\right.$ for some $\left.u \in V^{\prime}\right\}$. Let $\left(V^{\prime}, K^{\prime}\right)$ be an undirected graph. We call a subset $E^{\prime} \subset K^{\prime}$ a matching in $\left(V^{\prime}, K^{\prime}\right)$ if no two edges in $E^{\prime}$ share a common vertex. We define $\mathcal{M}^{\prime}\left(V^{\prime}, K^{\prime}\right)$ to be the set of matchings in $\left(V^{\prime}, K^{\prime}\right)$ and $\mathcal{M}^{\prime}\left(V^{\prime}\right)$ to be the set $\mathcal{M}^{\prime}\left(V^{\prime}, K_{V^{\prime}}^{\prime}\right)$ of matchings 
in the complete graph $\left(V^{\prime}, K_{V^{\prime}}^{\prime}\right)$. A matching $E^{\prime}$ in $\left(V^{\prime}, K^{\prime}\right)$ is said to be perfect if vertex $\left(E^{\prime}\right)=V^{\prime}$. We define $\mathcal{P}^{\prime}\left(V^{\prime}, K^{\prime}\right)$ to be the set of perfect matchings in $\left(V^{\prime}, K^{\prime}\right)$ and $\mathcal{P}^{\prime}\left(V^{\prime}\right)$ by $\mathcal{P}^{\prime}\left(V^{\prime}\right)=\mathcal{P}^{\prime}\left(V^{\prime}, K_{V^{\prime}}^{\prime}\right)$.

Next we consider directed graphs. A directed edge from $v$ to $w$ is denoted by $(v, u)$. For $v \neq u$, $(v, u) \neq(u, v)$. In the case of directed graphs, we also consider a directed self loop $(v, v)$. For a directed edge $e=(v, u)$, we respectively call $v$ and $u$ a starting and end points of $e$. For sets $W$ and $U$ of vertices, we define sets $K_{U}$ and $K_{W, U}$ of directed edges by $K_{W, U}=\{(v, u) \mid v \in W, u \in U\}$, $K_{U}=K_{U, U}=\{(v, u) \mid v, u \in U\}$. We call a pair $G=(V, E)$ of a finite set $V$ and a subset $E \subset K_{V}$ a directed graph. For a directed graph $G=(V, E)$, we define $\operatorname{start}(E)$ and end $(E)$ by

$$
\begin{aligned}
\operatorname{start}(E) & =\{v \in V \mid(v, u) \in E \text { for some } u \in V\}, \\
\operatorname{end}(E) & =\{u \in V \mid(v, u) \in E \text { for some } v \in V\} .
\end{aligned}
$$

For a directed graph $(V, K)$, we call a subset $E \subset K$ a matching in $(V, K)$ if $v \neq v^{\prime}$ and $u \neq u^{\prime}$ for any two distinct directed edges $(v, u)$ and $\left(v^{\prime}, u^{\prime}\right) \in E$. We define $\mathcal{M}(V, K)$ to be the set of matchings in $(V, K)$ and $\mathcal{M}(V)$ by $\mathcal{M}(V)=\mathcal{M}\left(V, K_{V}\right)$. A matching $E$ in $(V, E)$ is said to be perfect if $\operatorname{start}(E)=V$ and end $(E)=V$. We define $\mathcal{P}(V, K)$ to be the set of perfect matchings in $(V, K)$ and $\mathcal{P}(V)$ by $\mathcal{P}(V)=\mathcal{P}\left(V, K_{V}\right)$.

Remark 2.1 We can identify a directed graph $(V, E)$ with a bipartite graph $(\dot{V}, \ddot{V},\{\{\dot{v}, \ddot{u}\} \mid(v, u) \in E\})$. i.e., a graph whose edges connect a vertex in $\dot{V}$ to a vertex in $\ddot{V}$. Via this identification, an element in $\mathcal{M}(V, K)$ is identified with a matching in the bipartite graph. In this sense, we call an element in $\mathcal{M}(V, K)$ a matching in $(V, K)$.

\section{Weighted generating functions and moments}

First we consider undirected graphs to describe the moments of real Wishart distributions. For an undirected graph $\left(V^{\prime}, E^{\prime}\right)$ and variables $\boldsymbol{x}=\left(x_{i, j}\right)$, we define the weight monomial $\boldsymbol{x}^{E^{\prime}}$ by $\boldsymbol{x}^{E^{\prime}}=\prod_{\{v, u\} \in E^{\prime}} x_{v, u}$. If $x_{v, u}=x_{u, v}$ for $v, u \in V^{\prime}$, then the weight monomial $\boldsymbol{x}^{E^{\prime}}$ is well-defined. We define $E_{0}^{\prime}$ to be $\{\{\dot{1}, \ddot{1}\}, \ldots,\{\dot{n}, \ddot{n}\}\} \subset K_{\dot{V}, \ddot{V}}^{\prime}$. For $E^{\prime} \in \mathcal{M}^{\prime}\left(V^{\prime}\right)$, we define $\check{E}^{\prime}$ and len $\left(E^{\prime}\right)$ by

$$
\begin{aligned}
\check{E}^{\prime} & =\left\{\{v, u\} \in K_{V^{\prime} \backslash \operatorname{vertex}\left(E^{\prime}\right)}^{\prime} \mid \text { There exists a chain between } v \text { and } u \text { in } E^{\prime} \cup E_{0}^{\prime} .\right\} \subset K_{V^{\prime}}^{\prime} \\
\operatorname{len}\left(E^{\prime}\right) & \left.=\text { (the number of connected components in }\left(V^{\prime}, E^{\prime} \cup E_{0}^{\prime}\right)\right)-\left|\check{E}^{\prime}\right| .
\end{aligned}
$$

Remark 3.1 For $E^{\prime} \in \mathcal{M}^{\prime}\left(V^{\prime}\right), \check{E}^{\prime}$ can be defined as a subset of $K_{V^{\prime}}^{\prime}$ satisfying the following conditions:

- $\check{E}^{\prime} \in \mathcal{M}^{\prime}\left(V^{\prime}\right)$,

- $\check{E}^{\prime} \cap E^{\prime}=\emptyset$,

- $\check{E}^{\prime} \cup E^{\prime} \in \mathcal{P}^{\prime}\left(E^{\prime}\right)$,

- The number of connected components in $\left(V^{\prime}, E^{\prime} \cup E_{0}^{\prime}\right)$ equals the number of connected components in $\left(V^{\prime}, \check{E}^{\prime} \cup E^{\prime} \cup E_{0}^{\prime}\right)$. 
Remark 3.2 For $E^{\prime} \in \mathcal{M}^{\prime}\left(V^{\prime}\right)$, let us consider the undirected graph $\left(V^{\prime}, E^{\prime} \amalg E_{0}^{\prime}\right)$ with multiple edges. The connected components of $\left(V^{\prime}, E^{\prime} \amalg E_{0}^{\prime}\right)$ are chains and cycles without chords. The number of cycles in $\left(V^{\prime}, E^{\prime} \amalg E_{0}^{\prime}\right)$ equals len $\left(E^{\prime}\right)$. The vertices $V^{\prime} \backslash \operatorname{vertex}\left(E^{\prime}\right)$ which do not appear in $E^{\prime}$ are terminals of chains in $\left(V^{\prime}, E^{\prime} \amalg E_{0}^{\prime}\right)$. The set of pairs of terminals of chains in $\left(V^{\prime}, E^{\prime} \amalg E_{0}^{\prime}\right)$ equals $\check{E}^{\prime}$.

Definition 3.3 For a set $K^{\prime} \subset K_{V^{\prime}}^{\prime}$ of undirected edges, we define polynomials $\Phi_{K^{\prime}}^{\prime}$ and $\Psi_{K^{\prime}}^{\prime}$ by

$$
\Phi_{K^{\prime}}^{\prime}(t, \boldsymbol{x}, \boldsymbol{y})=\sum_{E^{\prime} \in \mathcal{M}^{\prime}\left(V^{\prime}, K^{\prime}\right)} t^{\operatorname{len}\left(E^{\prime}\right)} \boldsymbol{x}^{E^{\prime}} \boldsymbol{y}^{\check{E}^{\prime}}, \quad \Psi_{K^{\prime}}^{\prime}(t, \boldsymbol{x})=\sum_{E^{\prime} \in \mathcal{P}^{\prime}\left(V^{\prime}, K^{\prime}\right)} t^{\operatorname{len}\left(E^{\prime}\right)} \boldsymbol{x}^{E^{\prime}} .
$$

We also respectively define $\Phi^{\prime}(t, \boldsymbol{x}, \boldsymbol{y})$ and $\Psi^{\prime}(t, \boldsymbol{x})$ to be $\Phi_{K_{V^{\prime}}^{\prime}}^{\prime}(t, \boldsymbol{x}, \boldsymbol{y})$ and $\Psi_{K_{V^{\prime}}^{\prime}}^{\prime}(t, \boldsymbol{x})$.

Remark 3.4 By definition, $\Psi_{K^{\prime}}^{\prime}(t, \boldsymbol{x})=\Phi_{K^{\prime}}^{\prime}(t, \boldsymbol{x}, 0)$ for each $K^{\prime} \subset K_{V^{\prime}}^{\prime}$.

We have the following formula that describes the moments of the real noncentral Wishart distribution as the special values of the weighted generating function.

Theorem 3.5 Let $W=\left(w_{i, j}\right) \sim W_{p}(\nu, \Sigma, \Delta)$, namely, let $W$ be a random matrix distributed according to the real noncentral Wishart distribution $W_{p}(\nu, \Sigma, \Delta)$. Then

$\mathbb{E}\left[w_{1,2} w_{3,4} \cdots w_{2 n-1,2 n}\right]=\mathbb{E}\left[w_{i, i} w_{\dot{2}, \ddot{2}} \cdots w_{\dot{n}, \ddot{n}}\right]=\left.\Phi^{\prime}(t, \boldsymbol{x}, \boldsymbol{y})\right|_{t=\nu, x_{u, v}=\sigma_{u, v}, y_{u, v}=\delta_{u, v}}=\Phi^{\prime}(\nu, \Sigma, \Delta)$.

Corollary 3.6 For $W \sim W_{p}(\nu, \Sigma, \Delta)$

$$
\mathbb{E}\left[w_{i_{1}, i_{2}} w_{i_{3}, i_{4}} \cdots w_{i_{2 n-1}, i_{2 n}}\right]=\mathbb{E}\left[w_{i_{1}, i_{\ddot{1}}} w_{i_{\dot{2}}, i_{\ddot{2}}} \cdots w_{i_{\tilde{n}}, i_{\ddot{n}}}\right]=\left.\Phi^{\prime}(t, \boldsymbol{x}, \boldsymbol{y})\right|_{t=\nu, x_{u, v}=\sigma_{i_{u}, i_{v}}, y_{u, v}=\delta_{i_{u}, i_{v}}} .
$$

In the case where $\Delta=0, W_{p}(\nu, \Sigma, 0)$ is called the real central Wishart distribution and is denoted by $W_{p}(\nu, \Sigma)$. It follows from Remark 3.4 that the moments of the central real Wishart distribution are written as special values of $\Psi^{\prime}$.

Corollary 3.7 For $W=\left(w_{i, j}\right) \sim W_{p}(\nu, \Sigma)$

$$
\mathbb{E}\left[w_{1,2} w_{3,4} \cdots w_{2 n-1,2 n}\right]=\mathbb{E}\left[w_{i, \ddot{i}} w_{\dot{2}, \ddot{2}} \cdots w_{\dot{n}, \ddot{n}}\right]=\left.\Psi^{\prime}(t, \boldsymbol{x})\right|_{t=\nu, x_{u, v}=\sigma_{u, v}}=\Psi^{\prime}(\nu, \Sigma) .
$$

Corollary 3.8 For $W=\left(w_{i, j}\right) \sim W_{p}(\nu, \Sigma)$

$$
\mathbb{E}\left[w_{i_{1}, i_{2}} w_{i_{3}, i_{4}} \cdots w_{i_{2 n-1}, i_{2 n}}\right]=\mathbb{E}\left[w_{i_{1}, i_{\ddot{1}}} w_{i_{\dot{2}}, i_{\ddot{2}}} \cdots w_{i_{\dot{n}}, i_{\ddot{n}}}\right]=\left.\Psi^{\prime}(t, \boldsymbol{x})\right|_{t=\nu, x_{u, v}=\sigma_{i_{u}, i_{v}}} .
$$

Next we consider directed graphs to describe the moments of complex Wishart distributions. For a directed graph $(V, E)$ and variables $\boldsymbol{x}=\left(x_{i, j}\right)$, we define the weight monomial $\boldsymbol{x}^{E}$ by $\boldsymbol{x}^{E}=\prod_{(v, u) \in E} x_{v, u}$. Let $E \in \mathcal{M}(V)$. The pair $(V, E)$ is a directed graph whose connected components are directed chains and directed cycles without chords. We define len $(E)$ to be the number of cycles (and self loops) in $(V, E)$. The vertices $V \backslash \operatorname{start}(E)$ are the endpoints of the chains in $(V, E)$, while the vertices $V \backslash \operatorname{end}(E)$ are the start points of the chains in $(V, E)$. We define $\check{E}$ by

$$
\check{E}=\left\{(v, u) \in K_{V \backslash \operatorname{start}(E), V \backslash \operatorname{end}(E)} \mid \text { There exists a chain from } u \text { to } v \text { in } E \text {. }\right\} \subset K_{V} \text {. }
$$


Remark 3.9 For $E \in \mathcal{M}(V)$, $\check{E}$ can be defined as a subset of $K_{V}$ satisfying the following conditions:

- $\check{E} \in \mathcal{M}(V)$,

- $\check{E} \cap E=\emptyset$,

- $\check{E} \cup E \in \mathcal{P}(E)$,

- The number of connected components in $(V, E)$ equals the number of connected components in $(V, \check{E} \cup E)$.

Remark 3.10 For $E \in \mathcal{M}(V)$, we can also define len $(E)$ by

$$
\operatorname{len}(E)=(\text { the number of connected components in }(V, E))-|\check{E}| .
$$

Remark 3.11 We can identify $E \in \mathcal{P}(V)$ with the element $\sigma_{E}$ of the symmetric group $S_{n}$ such that $\sigma_{E}(i)=j$ for each $(i, j) \in E$. For each $E$, $\operatorname{len}(E)$ is the number of cycles of $\sigma_{E}$.

Definition 3.12 For a set $K \subset K_{V}$ of directed edges, we define polynomials $\Phi_{K}$ and $\Psi_{K}$ by

$$
\Phi_{K}(t, \boldsymbol{x}, \boldsymbol{y})=\sum_{E \in \mathcal{M}(V, K)} t^{\operatorname{len}(E)} \boldsymbol{x}^{E} \boldsymbol{y}^{\check{E}}, \quad \Psi_{K}(t, \boldsymbol{x})=\sum_{E \in \mathcal{P}(V, K)} t^{\operatorname{len}(E)} \boldsymbol{x}^{E} .
$$

We also respectively define $\Phi(t, \boldsymbol{x}, \boldsymbol{y})$ and $\Psi(t, \boldsymbol{x})$ to be $\Phi_{K_{V}}(t, \boldsymbol{x}, \boldsymbol{y})$ and $\Psi_{K_{V}}(t, \boldsymbol{x})$.

Remark 3.13 By definition, $\Psi_{K}(t, \boldsymbol{x})=\Phi_{K}(t, \boldsymbol{x}, 0)$ for each $K \subset K_{V}$.

We describe the moments of complex Wishart distributions as special values of the generating functions.

Theorem 3.14 Let $W=\left(w_{i, j}\right)$ be a random matrix distributed according to the complex noncentral Wishart distribution $C W_{p}(\nu, \Sigma, \Delta)$. Then

$$
\mathbb{E}\left[w_{1,2} w_{3,4} \cdots w_{2 n-1,2 n}\right]=\mathbb{E}\left[w_{1, \ddot{1}} w_{\dot{2}, \ddot{2}} \cdots w_{\dot{n}, \ddot{n}}\right]=\left.\Phi(t, \boldsymbol{x}, \boldsymbol{y})\right|_{t=\nu, x_{u, v}=\sigma_{\dot{u}, \ddot{v}}, y_{u, v}=\delta_{\dot{u}, \dot{v}}} .
$$

Corollary 3.15 For $W=\left(w_{i, j}\right) \sim C W_{p}(\nu, \Sigma, \Delta)$

$$
\mathbb{E}\left[w_{i_{1}, i_{2}} w_{i_{3}, i_{4}} \cdots w_{i_{2 n-1}, i_{2 n}}\right]=\mathbb{E}\left[w_{i_{1}, i_{\dot{1}}} w_{i_{\dot{2}}, i_{\ddot{2}}} \cdots w_{i_{\dot{n}}, i_{\ddot{n}}}\right]=\left.\Phi(t, \boldsymbol{x}, \boldsymbol{y})\right|_{t=\nu, x_{u, v}=\sigma_{i_{\dot{u}}, i_{\ddot{v}}}, y_{u, v}=\delta_{i_{\dot{u}}, i_{\ddot{v}}}} .
$$

By substituting 0 for $\Delta$ in the theorem, we have the following formula for the central complex case.

Corollary 3.16 For $W=\left(w_{i, j}\right) \sim C W_{p}(\nu, \Sigma)$

$$
\mathbb{E}\left[w_{1,2} w_{3,4} \cdots w_{2 n-1,2 n}\right]=\mathbb{E}\left[w_{i, \ddot{1}} w_{\dot{2}, \ddot{2}} \cdots w_{\dot{n}, \ddot{n}}\right]=\left.\Psi(t, \boldsymbol{x})\right|_{t=\nu, x_{u v}=\sigma_{\dot{u}, \ddot{v}}} .
$$

Corollary 3.17 For $W=\left(w_{i, j}\right) \sim C W_{p}(\nu, \Sigma)$

$$
\mathbb{E}\left[w_{i_{1}, i_{2}} w_{i_{3}, i_{4}} \cdots w_{i_{2 n-1}, i_{2 n}}\right]=\mathbb{E}\left[w_{i_{\mathrm{1}}, i_{\grave{1}}} w_{i_{\dot{2}}, i_{\ddot{2}}} \cdots w_{i_{\grave{n}}, i_{\ddot{n}}}\right]=\left.\Psi(t, \boldsymbol{x})\right|_{t=\nu, x_{u, v}=\sigma_{i_{\dot{u}}, i_{\ddot{v}}}} .
$$


Remark 3.18 For a square matrix $A=\left(a_{i j}\right)$, the $\alpha$-determinant (or $\alpha$-permanent) is defined by

$$
\operatorname{det}_{\alpha}(A)=\sum_{\sigma \in S_{n}} \alpha^{n-\operatorname{len}(\sigma)} a_{1, \sigma(1)} a_{2, \sigma(2)} \cdots a_{n, \sigma(n)}
$$

This polynomial is an $\alpha$-analogue of both the determinant and the permanent. Equivalently, the $\alpha$ determinant is nothing but the ordinary determinant and permanent for $\alpha=-1$ and 1 , respectively. (See also [16 [17].) Through the identification in Remark 3.10 we have $\alpha^{n} \Psi\left(\alpha^{-1}, A\right)=\operatorname{det}_{\alpha}(A)$. Moreover, by Corollary 3.16 the moments of the complex central Wishart distribution are expressed by $\alpha$-determinants.

In [12], Matsumoto introduced the $\alpha$-Pfaffian, which is defined by

$$
\operatorname{pf}_{\alpha}(A)=\sum_{E^{\prime} \in \mathcal{P}^{\prime}\left(V^{\prime}\right)}(-\alpha)^{n-\operatorname{len}\left(E^{\prime}\right)} \operatorname{sgn}\left(E^{\prime}\right) A^{E^{\prime}}
$$

for a skew-symmetric matrix $A$, where $\operatorname{sgn}\left(E^{\prime}\right) A^{E^{\prime}}$ is defined to be $\operatorname{sgn}(x) a_{x_{\dot{1}}, x_{\ddot{1}}} \cdots a_{x_{\dot{n}}, x_{\ddot{n}}}$ for $x \in S_{2 n}$ such that $E^{\prime}=\left\{\left\{x_{\dot{1}}, x_{\ddot{1}}\right\}, \ldots,\left\{x_{\dot{n}}, x_{\ddot{n}}\right\}\right\}$. Since $A$ is skew symmetric, $\operatorname{sgn}\left(E^{\prime}\right) A^{E^{\prime}}$ is independent from choices of $x \in S_{2 n}$. The $\alpha$-Pfaffian is an analogue of the Pfaffian. Equivalently, in the case when $\alpha=-1$, $\alpha$-Pfaffian $\mathrm{pf}_{-1}(A)$ is nothing but the ordinary Pfaffian $\operatorname{pf}(A)$, i.e., $\sum \operatorname{sgn}(x) a_{x_{\mathrm{i}} x_{\dot{1}}} \cdots a_{x_{\dot{n}} x_{\tilde{n}}}$.

Let us define the polynomial $\mathrm{hf}_{\alpha}(A)$ by

$$
\operatorname{hf}_{\alpha}(B)=\sum_{E^{\prime} \in \mathcal{P}^{\prime}\left(V^{\prime}\right)} \alpha^{n-\operatorname{len}\left(E^{\prime}\right)} B^{E^{\prime}}
$$

for a symmetric matrix $B$. The polynomial $\mathrm{hf}_{\alpha}(B)$ is an $\alpha$-analogue of the Hafnian. Equivalently, $\mathrm{hf}_{\alpha}(B)$ is the ordinary Hafnian $\mathrm{hf}(B)$, i.e., $\sum b_{x_{1} x_{1}} \cdots b_{x_{\dot{n}} x_{\ddot{n}}}$, for $\alpha=1$. By definition, $\operatorname{hf}_{\alpha}(B)=$ $\alpha^{n} \Psi^{\prime}\left(\alpha^{-1}, B\right)$. In this sense, the moments of the real central Wishart distributions are expressed by $\alpha$-Hafnians.

\section{Application}

\subsection{Relation between real and complex cases}

There exist bijections between directed graphs and undirected graphs which preserve the weight monomials in some special cases. These bijections induce equations between weighted generating functions of matchings. From the equations, we can obtain some formulas for the moments of complex and real Wishart distributions with special parameters.

\subsubsection{Prototypical case}

As in Remark 2.1, there exists a correspondence between directed graphs and bipartite graphs.

Lemma 4.1 The map $\mathcal{M}\left(V, K_{V}\right) \ni(u, v) \mapsto\{\dot{u}, \ddot{v}\} \in \mathcal{M}^{\prime}\left(V^{\prime}, K_{\dot{V}, \ddot{V}}^{\prime}\right)$ is a bijection. The map induces the bijection $\mathcal{P}\left(V, K_{V}\right) \ni(u, v) \mapsto\{\dot{u}, \ddot{v}\} \in \mathcal{P}^{\prime}\left(V^{\prime}, K_{\dot{V}, \ddot{V}}^{\prime}\right)$. 
These bijections imply $\Phi_{K_{V}}(t, \boldsymbol{x}, \boldsymbol{y})=\Phi_{K_{\dot{V}, \dot{V}}^{\prime}}^{\prime}(t, \boldsymbol{x}, \boldsymbol{y})$ and $\Psi_{K_{V}}(t, \boldsymbol{x})=\Psi_{K_{\dot{V}, \dot{V}}^{\prime}}^{\prime}(t, \boldsymbol{x})$. If $\Sigma^{\prime}=\left(\sigma_{u v}^{\prime}\right)$ and $\Delta^{\prime}=\left(\delta_{u v}^{\prime}\right)$ satisfy $\sigma_{u, v}^{\prime}=0$ and $\delta_{u, v}^{\prime}=0$ for $\{u, v\} \in K_{\dot{V}}^{\prime} \cup K_{\dot{V}}^{\prime}$, then

$$
\left.\Phi^{\prime}(t, \boldsymbol{x}, \boldsymbol{y})\right|_{t=\nu, x_{u, v}=\sigma_{u, v}^{\prime}, y_{u, v}=\delta_{u, v}^{\prime}}=\left.\Phi_{K_{\dot{V}, \dot{V}}^{\prime}}^{\prime}(t, \boldsymbol{x}, \boldsymbol{y})\right|_{t=\nu, x_{u, v}=\sigma_{u, v}^{\prime}, y_{u, v}=\delta_{u, v}^{\prime}} .
$$

If $\Sigma=\left(\sigma_{u v}\right)$ and $\Delta=\left(\delta_{u v}\right)$ satisfy $\sigma_{\dot{u}, \ddot{v}}=\sigma_{u, v}^{\prime}$ and $\delta_{\dot{u}, \dot{v}}=\delta_{u, v}^{\prime}$ for $u, v \in V$, then the equation implies

$$
\left.\Phi(t, \boldsymbol{x}, \boldsymbol{y})\right|_{t=\nu, x_{u, v}=\sigma_{\dot{u}, \dot{v}}, y_{u, v}=\delta_{\dot{u}, \dot{v}}}=\left.\Phi^{\prime}(t, \boldsymbol{x}, \boldsymbol{y})\right|_{t=\nu, x_{u, v}=\sigma_{u, v}, y_{u, v}=\delta_{u, v}} .
$$

Hence we have the following:

Propsition 4.2 Let $\Sigma^{\prime}=\left(\sigma_{u, v}^{\prime}\right), \Delta^{\prime}=\left(\delta_{u, v}^{\prime}\right), \Sigma=\left(\sigma_{u, v}\right)$ and $\Delta=\left(\delta_{u, v}\right)$ satisfy $\sigma_{u, v}^{\prime}=0, \delta_{u, v}^{\prime}=0$ for $\{u, v\} \in K_{\dot{V}}^{\prime} \cup K_{\ddot{V}}^{\prime}$, and $\sigma_{\dot{u}, \ddot{v}}=\sigma_{\dot{u}, \dot{v}}^{\prime}, \delta_{\dot{u}, \ddot{v}}=\delta_{\dot{u}, \dot{v}}^{\prime}$ for $u, v \in V$. For $W=\left(w_{u, v}\right) \sim C W_{p}(\nu, \Sigma, \Delta)$ and $W^{\prime}=\left(w_{u, v}^{\prime}\right) \sim W_{p}\left(\nu, \Sigma^{\prime}, \Delta^{\prime}\right), \mathbb{E}\left[w_{i, \ddot{i}} \cdots w_{\dot{n}, \ddot{n}}\right]=\mathbb{E}\left[w_{\dot{1}, i}^{\prime} \cdots w_{\dot{n}, \ddot{n}}^{\prime}\right]$.

\subsubsection{Central case}

Next we consider the central Wishart distribution. In this case, we may consider only perfect matchings. We define $\widetilde{\mathcal{P}}^{\prime}\left(V^{\prime}\right)$ and $\widetilde{\mathcal{P}}(V)$ by

$$
\widetilde{\mathcal{P}}^{\prime}\left(V^{\prime}\right)=\left\{\left(E^{\prime}, \omega^{\prime}\right) \mid \begin{array}{c}
E^{\prime} \in \mathcal{P}^{\prime}\left(V^{\prime}\right), \\
\omega^{\prime}:\left\{\operatorname{cyclesin}\left(V^{\prime}, E \amalg E_{0}\right)\right\} \rightarrow\{ \pm 1\}
\end{array}\right\}, \quad \widetilde{\mathcal{P}}(V)=\left\{(E, \omega) \mid \begin{array}{c}
E \in \mathcal{P}(V), \\
\omega: E \rightarrow\{ \pm 1\}
\end{array}\right\} .
$$

Lemma 4.3 There exists a bijection between $\widetilde{\mathcal{P}}^{\prime}\left(V^{\prime}\right)$ and $\widetilde{\mathcal{P}}(V)$.

We shall give a bijection $\psi$ between $\widetilde{\mathcal{P}}^{\prime}\left(V^{\prime}\right)$ and $\widetilde{\mathcal{P}}(V)$ in Section 4.1 .4 The bijection preserves the weight monomials, equivalently, $t^{\operatorname{len}(E)} \boldsymbol{x}^{E}=t^{\operatorname{len}\left(E^{\prime}\right)}\left(\boldsymbol{x}^{\prime}\right)^{E^{\prime}}$ for elements $E^{\prime} \in \mathcal{P}^{\prime}(V)$ corresponding to $E \in \mathcal{P}(V)$, in the case when $\boldsymbol{x}=\left(x_{u, v}\right)$ and $\boldsymbol{x}^{\prime}=\left(x_{u, v}^{\prime}\right)$ satisfy $x_{u^{\prime}, v^{\prime}}^{\prime}=x_{u, v}$ for any $u, v \in V$ and any

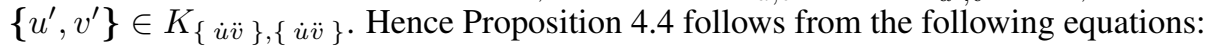

$$
\begin{aligned}
2^{n} \Psi(t, \boldsymbol{x}) & =2^{n} \sum_{E \in \mathcal{P}(V)} t^{\operatorname{len}(E)} \boldsymbol{x}^{E}=\sum_{E \in \widetilde{\mathcal{P}}(V)} t^{\operatorname{len}(E)} \boldsymbol{x}^{E} \\
\Psi^{\prime}(2 t, \boldsymbol{x}) & =2^{n} \sum_{E^{\prime} \in \mathcal{P}^{\prime}\left(V^{\prime}\right)}(2 t)^{\operatorname{len}\left(E^{\prime}\right)} \boldsymbol{x}^{E^{\prime}}=\sum_{E^{\prime} \in \widetilde{\mathcal{P}}\left(V^{\prime}\right)} t^{\operatorname{len}\left(E^{\prime}\right)} \boldsymbol{x}^{E^{\prime}} .
\end{aligned}
$$

Propsition 4.4 Let $\Sigma=\left(\sigma_{u, v}\right)$ and $\Sigma^{\prime}=\left(\sigma_{u, v}^{\prime}\right)$ satisfy $\sigma_{u^{\prime}, v^{\prime}}^{\prime}=\sigma_{u, v}$ for any $u, v \in V$ and any $\left\{u^{\prime}, v^{\prime}\right\} \in K_{\{\dot{u} \ddot{v}\},\{\dot{u} \ddot{v}\} \text {. Then }}$

$$
\left.2^{n} \Psi(t, \boldsymbol{x})\right|_{t=\nu, x_{u, v}=\sigma_{u, v}}=\left.\Psi^{\prime}(2 t, \boldsymbol{x})\right|_{t=\nu, x_{u, v}=\sigma_{u, v}^{\prime}} .
$$

Corollary 4.5 Let $\Sigma=\left(\sigma_{u, v}\right)$ and $\Sigma^{\prime}=\left(\sigma_{u, v}^{\prime}\right)$ satisfy $\sigma_{u^{\prime}, v^{\prime}}^{\prime}=\sigma_{u, v}$ for any $u, v \in V$ and any $\left\{u^{\prime}, v^{\prime}\right\} \in$ $K_{\{\dot{u} \ddot{v}\},\{\dot{u} \ddot{v}\}}$. For $W=\left(w_{u, v}\right) \sim C W_{p}(\nu, \Sigma)$ and $W^{\prime}=\left(w_{u, v}^{\prime}\right) \sim W_{p}\left(\nu, \Sigma^{\prime}\right), \mathbb{E}\left[w_{i, \ddot{1}} \cdots w_{\dot{n}, \ddot{n}}\right]=$ $\mathbb{E}\left[w_{i, i}^{\prime} \cdots w_{\dot{n}, \ddot{n}}^{\prime}\right]$. 


\subsubsection{Noncentral case}

Next we consider the noncentral Wishart distribution. In this case, we consider all matchings in complete graphs. We define $\widetilde{\mathcal{M}}(V)$ and $\widetilde{\mathcal{M}^{\prime}}\left(V^{\prime}\right)$ by

$$
\widetilde{\mathcal{M}^{\prime}}\left(V^{\prime}\right)=\left\{\left(E^{\prime}, \omega^{\prime}\right) \mid \begin{array}{c}
E^{\prime} \in \mathcal{M}^{\prime}\left(V^{\prime}\right), \\
\omega^{\prime}:\left\{\operatorname{cyclesin}\left(V^{\prime}, E^{\prime} \cup E_{0}^{\prime}\right)\right\} \rightarrow\{ \pm 1\}
\end{array}\right\}, \quad \widetilde{\mathcal{M}}(V)=\left\{\begin{array}{l|l}
(E, \omega) \mid \begin{array}{c}
E \in \mathcal{M}(V), \\
\omega: E \rightarrow\{ \pm 1\}
\end{array}
\end{array}\right\} .
$$

Lemma 4.6 There exists a bijection between $\widetilde{\mathcal{M}}(V)$ and $\widetilde{\mathcal{M}^{\prime}}\left(V^{\prime}\right)$.

We shall give a bijection between $\widetilde{\mathcal{P}}^{\prime}\left(V^{\prime}\right)$ and $\widetilde{\mathcal{P}}(V)$ in Section 4.1 .4 The bijection preserves the weight monomial in a special case. Hence Proposition 4.7 follows from the following equations:

$$
\begin{aligned}
\Phi^{\prime}(2 t, \boldsymbol{x}, \boldsymbol{y}) & =\sum_{E^{\prime} \in \mathcal{M}^{\prime}\left(V^{\prime}\right)}(2 t)^{\operatorname{len}\left(E^{\prime}\right)} \boldsymbol{x}^{E^{\prime}} \boldsymbol{y}^{\check{E^{\prime}}}=\sum_{\left(E^{\prime}, \omega^{\prime}\right) \in \widetilde{\mathcal{M}}^{\prime}\left(V^{\prime}\right)} t^{\operatorname{len}\left(E^{\prime}\right)} \boldsymbol{x}^{E^{\prime}} \boldsymbol{y}^{\check{E}^{\prime}}, \\
\Phi(t, 2 \boldsymbol{x}, \boldsymbol{y}) & =\sum_{E \in \mathcal{M}(V)} t^{\operatorname{len}(E)}(2 \boldsymbol{x})^{E} \boldsymbol{y}^{\check{E}}=\sum_{(E, \omega) \in \widetilde{\mathcal{M}}(V)} t^{\operatorname{len}(E)} \boldsymbol{x}^{E} \boldsymbol{y}^{\check{E}},
\end{aligned}
$$

where $2 \boldsymbol{x}=\left(2 x_{u, v}\right)$

Propsition 4.7 Let $\Sigma=\left(\sigma_{u, v}\right), \Delta=\left(\delta_{u, v}\right), \Sigma^{\prime}=\left(\sigma_{u, v}^{\prime}\right)$ and $\Delta^{\prime}=\left(\delta_{u, v}^{\prime}\right)$ satisfy $\sigma_{u^{\prime}, v^{\prime}}^{\prime}=\sigma_{u, v}$

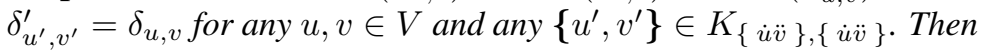

$$
\left.\Psi(t, 2 \boldsymbol{x}, \boldsymbol{y})\right|_{t=\nu, x_{u, v}=\sigma_{\dot{u}, \ddot{v}}, y_{u, v}=\delta_{\dot{u}, \dot{v}}}=\left.\Psi^{\prime}(2 t, \boldsymbol{x}, \boldsymbol{y})\right|_{t=\nu, x_{u, v}=\sigma_{u, v}, y_{u, v}=\delta_{u, v}} .
$$

Corollary 4.8 Let $\Sigma=\left(\sigma_{u, v}\right), \Delta=\left(\delta_{u, v}\right), \Sigma^{\prime}=\left(\sigma_{u, v}^{\prime}\right)$ and $\Delta^{\prime}=\left(\delta_{u, v}^{\prime}\right)$ satisfy $\sigma_{u^{\prime}, v^{\prime}}^{\prime}=\sigma_{u, v} \delta_{u^{\prime}, v^{\prime}}^{\prime}=$ $\delta_{u, v}$ for any $u, v \in V$ and any $\left.\left\{u^{\prime}, v^{\prime}\right\} \in K_{\{\dot{u} u}\right\},\{\dot{u} \ddot{u}\}$. For $W=\left(w_{u, v}\right) \sim C W_{p}(\nu, 2 \Sigma, \Delta)$ and $W^{\prime}=\left(w_{u, v}^{\prime}\right) \sim W_{p}\left(2 \nu, \Sigma^{\prime}, \Delta^{\prime}\right), \mathbb{E}\left[w_{i, i} \cdots w_{\dot{n}, \ddot{n}}\right]=\mathbb{E}\left[w_{\dot{1}, i}^{\prime} \cdots w_{\dot{n}, \ddot{n}}^{\prime}\right]$.

\subsubsection{Construction of Bijections}

Here we construct bijections to prove Lemmas 4.3 and 4.6 First we construct a bijection $\psi$ from $\widetilde{\mathcal{P}}(V)$ to $\widetilde{\mathcal{P}}^{\prime}\left(V^{\prime}\right)$. To define the bijection, we define the following map. For $(E, \omega) \in \widetilde{\mathcal{P}}(V)$, let $h_{E, \omega}$ and $h_{E, \omega}^{\prime}$ be maps from $V$ to $V^{\prime}$ defined by

$$
\begin{aligned}
& h_{E, \omega}(v)= \begin{cases}\dot{v} & \text { if } \omega((u, v))=1 \text { for some }(u, v) \in E, \\
\ddot{v} & \text { otherwise }\end{cases} \\
& h_{E, \omega}^{\prime}(v)= \begin{cases}\ddot{v} & \text { if } \omega((u, v))=1 \text { for some }(u, v) \in E, \\
\dot{v} & \text { otherwise }\end{cases}
\end{aligned}
$$

Remark 4.9 For $(E, \omega) \in \widetilde{\mathcal{P}}(V)$ and $v \in V,\left\{h_{E, \omega}(v), h_{E, \omega}^{\prime}(v)\right\} \in E_{0}^{\prime}$.

First we construct $E^{\prime} \in \mathcal{P}^{\prime}\left(V^{\prime}\right)$ for each $(E, \omega) \in \widetilde{\mathcal{P}}(V)$. For each $(E, \omega) \in \widetilde{\mathcal{P}}(V)$, we define a surjection $\psi_{E, \omega}: E \rightarrow K_{V^{\prime}}^{\prime}$, and then we define $E^{\prime}$ to be the image $\psi_{E, \omega}(E)$. Let $\left(v_{1}, v_{2}\right),\left(v_{2}, v_{3}\right), \ldots,\left(v_{k-1}, v_{k}\right)$, 
$\left(v_{k}, v_{1}\right)$ be a cycle in $E$ such that $v_{1}=\min \left\{v_{1}, \ldots, v_{k}\right\}$. For each directed edge in the cycle, we define $\psi_{E, \omega}$ by

$$
\begin{aligned}
\psi_{E, \omega}\left(\left(v_{1}, v_{2}\right)\right) & =\left\{\dot{v}_{1}, h_{E, \omega}\left(v_{2}\right)\right\}, \\
\psi_{E, \omega}\left(\left(v_{i}, v_{i+1}\right)\right) & =\left\{h_{E, \omega}^{\prime}\left(v_{i+1}\right), h_{E, \omega}\left(v_{i+1}\right)\right\} \quad(\text { for } i=2, \ldots, k-1), \\
\psi_{E, \omega}\left(\left(v_{k}, v_{1}\right)\right) & =\left\{h_{E, \omega}^{\prime}\left(v_{k}\right), \ddot{v}_{1}\right\} .
\end{aligned}
$$

Then the image of the cycle forms a cycle $C^{\prime}$ in the undirected graph $E^{\prime} \amalg E_{0}^{\prime}$. For the cycle $C^{\prime}$, we define $\omega^{\prime}\left(C^{\prime}\right)$ to be $\omega\left(\left(v_{k}, v_{1}\right)\right)$.

Remark 4.10 It is easy to construct the inverse map of $\psi$, which implies that $\psi$ is bijective.

Remark 4.11 This correspondence $\psi$ is equivalent to the one in [4], which is described in more algebraic terms. Let $S_{2 m}$ be the $2 m$-th symmetric group, and let $B_{m}=S_{m} \prec \mathbb{Z} / 2 \mathbb{Z}$ the hyperoctehedral group, i.e., the subgroup of the permutations $\pi \in S_{2 m}$ such that $|\pi(\dot{n})-\pi(\ddot{n})|=1$ for all $n=1, \ldots, m$. For $g B_{m} \in S_{2 m} / B_{m}$, we can define $E_{g B_{m}}$ by $E_{g B_{m}}=\{\{g(\dot{n}), g(\ddot{n})\} \mid n=1, \ldots m\} \in \mathcal{P}(V)$, and we can identify elements $g B_{m} \in S_{2 m} / B_{m}$ with perfect matchings in $\left(V, K_{V}\right)$. Through this identification, the correspondence in Section 4 of [4] is equivalent to ours.

Next we construct a bijection $\varphi$ from $\widetilde{\mathcal{M}}(V)$ to $\widetilde{\mathcal{M}}\left(V^{\prime}\right)$. For each $(E, \omega) \in \widetilde{\mathcal{M}}(V)$, we shall define $\left(E^{\prime}, \omega^{\prime}\right) \in \widetilde{\mathcal{M}}(V)$. For each cycle in $E$, we construct undirected edges and $\omega^{\prime}$ in the same manner as $\psi$. To define the undirected edges corresponding to chains, we define $t_{E, \omega}$ and $t_{E, \omega}^{\prime}$ by

$$
\begin{aligned}
& t_{E, \omega}(v)= \begin{cases}\dot{v} & \text { if } \omega((u, v))=1 \text { for some }(v, u) \in E, \\
\ddot{v} & \text { otherwise, }\end{cases} \\
& t_{E, \omega}^{\prime}(v)= \begin{cases}\ddot{v} & \text { if } \omega((u, v))=1 \text { for some }(v, u) \in E, \\
\dot{v} & \text { otherwise. }\end{cases}
\end{aligned}
$$

Let $\left(v_{1}, v_{2}\right),\left(v_{2}, v_{3}\right), \ldots,\left(v_{k-1}, v_{k}\right)$ be a maximal chain in $E$. If $v_{1}<v_{k}$, then we define $\varphi_{E, \omega}$ by

$$
\begin{aligned}
\varphi_{E, \omega}\left(\left(v_{1}, v_{2}\right)\right) & =\left\{\dot{v}_{1}, h_{E, \omega}\left(v_{2}\right)\right\}, \\
\varphi_{E, \omega}\left(\left(v_{i}, v_{i+1}\right)\right) & =\left\{h_{E, \omega}^{\prime}\left(v_{i}\right), h_{E, \omega}\left(v_{i+1}\right)\right\} \quad(\text { for } i=2, \ldots, k-1) .
\end{aligned}
$$

If $v_{1}>v_{k}$, then we define $\varphi_{E, \omega}$ by

$$
\begin{aligned}
\varphi_{E, \omega}\left(\left(v_{k-1}, v_{k}\right)\right) & =\left\{t_{E, \omega}\left(k_{k-1}\right), \ddot{v}_{k}\right\} \\
\varphi_{E, \omega}\left(\left(v_{i-1}, v_{i}\right)\right) & =\left\{t_{E, \omega}\left(v_{i-1}\right), t_{E, \omega}^{\prime}\left(v_{i}\right)\right\} \quad(\text { for } i=k-1, \ldots, 2) .
\end{aligned}
$$

Then the image of the maximal chains forms a maximal chain in the undirected graph $E^{\prime} \amalg E_{0}^{\prime}$.

Remark 4.12 It is easy to construct the inverse map of $\varphi$, which implies $\varphi$ is bijective.

\subsection{Noncentral chi-square distribution}

In this section, we consider the Wishart distributions for a special parameter, which is linked with the noncentral chi-square distributions. 
Propsition 4.13 Let $\sigma_{u, v}=\sigma, \delta_{u, v}=\delta$. For $W=\left(w_{u, v}\right) \sim C W_{p}(\nu, \Sigma, \Delta)$,

$$
\mathbb{E}\left[w_{\dot{1}, \dot{1}} w_{\dot{2}, \ddot{2}} \cdots w_{\dot{n}, \ddot{n}}\right]=\sum_{m=0}^{n} \sum_{l} g_{l m n} \nu^{l} \sigma^{m} \delta^{n-m}
$$

where $g_{\text {lmn }}$ is the number of $E \subset K_{V}$ such that $\operatorname{len}(E)=l,|E|=m$ and $|\check{E}|=n-m$.

Corollary 4.14 For the noncentral complex chi-square distribution $\chi_{\nu}^{2}(\delta)$ with $\nu$ degrees of freedom and the noncentrality parameter $\delta$, its $n$-th moment $\mathbb{E}\left(w^{n}\right)$ is given as follows:

$$
\mathbb{E}\left[w^{n}\right]=\sum_{m=0}^{n} \sum_{l} g_{l m n} \nu^{l} \delta^{n-m} .
$$

In the case where we add a new directed edge whose staring point is a fixed vertex, we have just one choice of end-points that increase the number of cycles. Hence we obtain Lemma 4.15.

Lemma 4.15 Let $0 \leq m \leq n$. Then the generating function $G_{m n}(t)$ of $g_{l m n}$ with respect to the number l of cycles satisfies

$$
G_{m n}(t)=\sum_{l \geq 0} g_{l m n} t^{l}=\left(\begin{array}{c}
n \\
m
\end{array}\right) \prod_{i=1}^{m}(t+n-i)
$$

We also obtain the following corollary, which is well-known expression for the noncentral chi-square distribution (e.g. [5])

Corollary 4.16 For the $n$-th moment $\mathbb{E}\left(w^{n}\right)$ of the noncentral chi-square distribution $\chi_{\nu}^{2}(\delta)$ with $\nu$ degrees of freedom and the noncentrality parameter $\delta$,

$$
\mathbb{E}\left[w^{n}\right]=\sum_{m=0}^{n} \sum_{l} g_{l m n} \nu^{l} \delta^{n-m}=\sum_{m=0}^{n} G_{m n}(\nu) \delta^{n-m}=\sum_{m=0}^{n}\left(\begin{array}{c}
n \\
m
\end{array}\right) \delta^{n-m} \prod_{i=1}^{m}(\nu+n-i) .
$$

Remark 4.17 The numbers $s_{n}(m, l)$ defined by the following generating function are called the noncentral Stirling numbers of the first kind:

$$
\sum_{l} s_{n}(m, l) t^{l}=\prod_{i=1}^{m}(t+n-i)
$$

If $m=n$, then $s_{n}(m, l)$ is the Stirling number of the first kind. Lemma 4.15 implies that $g_{l m n}=$ $\left(\begin{array}{c}n \\ m\end{array}\right) s_{n}(m, l)$. Equivalently, we can explicitly describe the moments of the noncentral chi-square distribution $\chi_{\nu}^{2}(\delta)$ with the noncentral Stirling numbers. Koutras pointed out that moments of some noncentral distributions are described with the noncentral Stirling numbers of the first kind [7].

Next consider the real case.

Propsition 4.18 Let $\sigma_{u, v}=\sigma, \delta_{u, v}=\delta$. For $W=\left(w_{u, v}\right) \sim W_{p}(\nu, \Sigma, \Delta)$,

$$
\mathbb{E}\left[w_{\dot{1}, \dot{1}} w_{\dot{2}, \ddot{2}} \cdots w_{\dot{n}, \ddot{n}}\right]=\sum_{m=0}^{n} \sum_{l} g_{l m n}^{\prime} \nu^{l} \sigma^{m} \delta^{n-m},
$$

where $g_{l m n}^{\prime}$ is the number of $E^{\prime} \subset K_{V^{\prime}}^{\prime}$ such that $\operatorname{len}\left(E^{\prime}\right)=l,\left|E^{\prime}\right|=m$ and $\left|\check{E}^{\prime}\right|=n-m$. 
Corollary 4.19 For the noncentral chi-square distribution $\chi_{\nu}^{2}(\delta)$ with $\nu$ degrees of freedom and the noncentrality parameter $\delta$, its $n$-th moment $\mathbb{E}\left(w^{n}\right)$ is given as:

$$
\mathbb{E}\left[w^{n}\right]=\sum_{m=0}^{n} \sum_{l} g_{l m n}^{\prime} \nu^{l} \delta^{n-m}
$$

where $g_{l m n}^{\prime}$ is the number of $E^{\prime} \subset K_{V^{\prime}}^{\prime}$ such that $\operatorname{len}\left(E^{\prime}\right)=l,\left|E^{\prime}\right|=m$ and $\left|\check{E}^{\prime}\right|=n-m$.

Proposition 4.7 and Lemma 4.15 imply Lemma 4.20

Lemma 4.20 Let $0 \leq m \leq n, n \leq 0$. Then the generating function $G_{m n}^{\prime}(t)$ of $g_{l m n}^{\prime}$ with respect to the number $l$ of cycles satisfies

$$
G_{m n}^{\prime}(t)=\sum_{l \geq 0} g_{l m n}^{\prime} t^{l}=\left(\begin{array}{c}
n \\
m
\end{array}\right) \prod_{i=1}^{m}(t+2(n-i)) .
$$

Corollary 4.21 For the $n$-th moment $\mathbb{E}\left(w^{n}\right)$ of the noncentral chi-square distribution $\chi_{\nu}^{2}(\delta)$ with $\nu$ degrees of freedom and the noncentrality parameter $\delta$,

$$
\mathbb{E}\left[w^{n}\right]=\sum_{m=0}^{n} \sum_{l} g_{l m n}^{\prime} \nu^{l} \delta^{n-m}=\sum_{m=0}^{n} G_{m n}^{\prime}(\nu) \delta^{n-m}=\sum_{m=0}^{n}\left(\begin{array}{c}
n \\
m
\end{array}\right) \delta^{n-m} \prod_{i=1}^{m}(\nu+2(n-i)) .
$$

\subsection{Bivariate chi-square distribution}

We can explicitly describe the moments of Wishart distributions by enumerating the matchings satisfying some conditions. For example, in Proposition 4.23, we obtain the description of the moments of the bivariate real chi-square distribution, which was introduced by Kibble [6]. The formulas imply formulas for the complex distribution by Proposition 4.7. See [8] for details and other applications.

Propsition 4.22 Let $\Sigma=\left(\sigma_{u v}\right)$ and $\Delta=\left(\delta_{u v}\right)$ satisfy

$$
\sigma_{u, v}=\left\{\begin{array}{ll}
1 & (u, v \leq 2 b \text { or } 2 b+1 \leq u, v), \\
\rho & \text { (otherwise }),
\end{array} \quad \delta_{u, v}=0 .\right.
$$

For a random matrix $W=\left(w_{u, v}\right) \sim W_{b+c}(\nu, \Sigma, \Delta)$,

$$
\begin{aligned}
\mathbb{E} & {\left[w_{i, \ddot{1}} \cdots w_{\dot{b}, \ddot{b}} \cdot w_{(b \dot{+} 1),(b \ddot{+} 1)} \cdots w_{(b \dot{+}),(b \ddot{+} c)}\right] } \\
& =\sum_{a=0}^{\min (b, c)} \rho^{2 a} \frac{2^{a} b ! c !}{(b-a) !(c-a) ! a !} \prod_{i=1}^{a}(\nu+a(a-i)) \prod_{i=1}^{b-a}(\nu+a(b-i)) \prod_{i=1}^{c-a}(\nu+a(c-i)) .
\end{aligned}
$$

Propsition 4.23 Let $\Sigma=\left(\begin{array}{ll}1 & \rho \\ \rho & 1\end{array}\right)$, and $W=\left(w_{u, v}\right) \sim W_{2}(\nu, \Sigma)$. For $b, c \in \mathbb{Z}_{\geq 0}$,

$$
\mathbb{E}\left[w_{1,1}^{b} w_{2,2}^{c}\right]=\sum_{a=0}^{\min (b, c)} \rho^{2 a} \frac{2^{a} b ! c !}{(b-a) !(c-a) ! a !} \prod_{i=1}^{a}(\nu+2(a-i)) \prod_{i=1}^{b-a}(\nu+2(b-i)) \prod_{i=1}^{c-a}(\nu+2(c-i)) .
$$

Remark 4.24 In [14], Nadarajah and Kotz derived another expression for $\mathbb{E}\left[w_{1,1}^{b} w_{2,2}^{c}\right]$ with the Jacobi polynomials. 


\section{References}

[1] Bai, Z. D. (1999). Methodologies in spectral analysis of large dimensional random matrices, A review. Statist. Sinica, 9, 611-677.

[2] Goodman, N. R. (1963). Statistical analysis based on a certain multivariate complex Gaussian distribution (An introduction). Ann. Math. Statist., 34, 152-177.

[3] Graczyk, P., Letac, G. and Massam, H. (2003). The complex Wishart distribution and the symmetric groups. Ann. Statist., 31, 287-309.

[4] Graczyk, P., Letac, G. and Massam, H. (2005). The hyperoctahedral group, symmetric group representations and the moments of the real Wishart distribution. J. Theor. Probab., 18, 1-42.

[5] Johnson, N. L., Kotz, S. and Balakrishnan, N. (1995). Continuous Univariate Distributions, Vol. 2, 2nd ed. Wiley-Interscience.

[6] Kibble, W. F. (1941). A two-variate gamma type distribution. Sankhya, 5A, 137-150.

[7] Koutras, M. (1982). Noncentral Stirling numbers and some applications. Discrete Math., 42, 73-89.

[8] Kuriki, S. and Numata, Y. (2010). Graph representations for moments of noncentral Wishart distributions and their applications. Annals of the Institute of Statistical Mathematics, 62 4, 645-672.

[9] Letac, G. and Massam, H. (2008). The noncentral Wishart as an exponential family, and its moments. J. Multivariate Anal., 99, 1393-1417.

[10] Lu, I-L. and Richards, D. St. P. (2001). MacMahon's master theorem, representation theory, and moments of Wishart distributions. Adv. Appl. Math., 27, 531-547.

[11] Maiwald, D. and Kraus, D. (2000). Calculation of moments of complex Wishart and complex inverse Wishart distributed matrices. IEE Proc.-Radar, Sonar Navig, 147, 162-168.

[12] Matsumoto, S. (2005) $\alpha$-Pfaffian, pfaffian point process and shifted Schur measure. Linear Algebra and its Applications, 403, 369-398.

[13] Muirhead, R. J. (1982). Aspects of Multivariate Statistical Theory. John Wiley \& Sons

[14] Nadarajah, S. and Kotz, S. (2006). Product moments of Kibble's bivariate gamma distribution. Circuits Systems Signal Process., 25, 567-570.

[15] Takemura, A. (1991). Foundations of Multivariate Statistical Inference (in Japanese). Kyoritsu Shuppan.

[16] Vere-Jones, D. (1988). A generalization of permanents and determinants. Linear Algebra Appl., 111, 119-124.

[17] Vere-Jones, D. (1997). Alpha-permanents and their applications to multivariate gamma, negative binomial and ordinary binomial distributions. New Zealand J. Math., 26, 125-149.

[18] Wishart, J. (1928). The generalised product moment distribution in samples from a normal multivariate population. Biometrika, 20A, 32-52. 